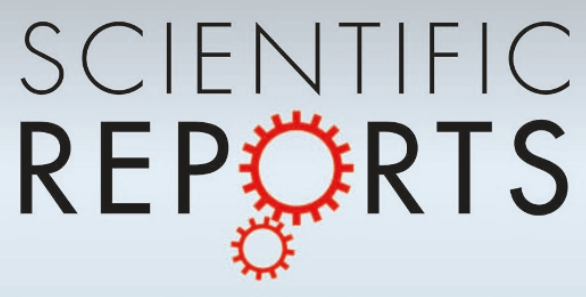

OPEN

SUBJECT AREAS:

ELECTRONIC DEVICES

ELECTRONIC PROPERTIES AND

MATERIALS

SOLAR ENERGY AND

PHOTOVOLTAIC

TECHNOLOGY

SOLAR CELLS

Received

17 September 2014

Accepted

28 November 2014

Published

9 January 2015

Correspondence and requests for materials should be addressed to J.J. (jhjeong@kist.re.kr)

\section{Characterization of efficiency-limiting} resistance losses in monolithically integrated $\mathrm{Cu}(\mathrm{In}, \mathrm{Ga}) \mathrm{Se}_{2}$ solar modules

\author{
Ju-Heon Yoon', Jong-Keuk Park', Won Mok Kim', JinWoo Lee², Hisun Pak² \& Jeung-hyun Jeong' \\ ${ }^{1}$ Korea Institute of Science and Technology (KIST), Hwarangno 14-gil 5, Seongbuk-gu, Seoul 136-791, Korea, ${ }^{2}$ LG Innotek R\&D \\ Center, 55, Hanyang Daehak-ro, Sanggrok-gu, Ansan, Gyeonggi, 426-791, Korea.
}

The cell-to-module efficiency gap in $\mathrm{Cu}(\mathrm{In}, \mathrm{Ga}) \mathrm{Se}_{2}$ (CIGS) monolithically integrated solar modules is enhanced by contact resistance between the Al-doped $\mathrm{ZnO}$ (AZO) and Mo back contact layers, the P2 contact, which connects adjacent cells. The present work evaluated the P2 contact resistance, in addition to the TCO resistance, using an embedded transmission line structure in a commercial-grade module without using special sample fabrication methods. The AZO layers between cells were not scribed; instead, the CIGS/ $\mathrm{CdS} / \mathrm{i}-\mathrm{ZnO} / \mathrm{AZO}$ device was patterned in a long stripe to permit measurement of the Mo electrode pair resistance over current paths through two P2 contacts (Mo/AZO) and along the AZO layer. The intercept and slope of the resistance as a function of the electrode interval yielded the P2 contact resistance and the TCO resistance, respectively. Calibration of the parasitic resistances is discussed as a method of improving the measurement accuracy. The contribution of the P2 contact resistance to the series resistance was comparable to that of the TCO resistance, and its origin was attributed to remnant $\mathrm{MoSe}_{2}$ phases in the P2 region, as verified by transmission electron microscopy.

C $\mathrm{u}(\mathrm{In}, \mathrm{Ga}) \mathrm{Se}_{2}$ (CIGS) thin film solar cells have attracted much interest as a renewable energy source because they may provide low-cost high-efficiency commercial-grade modules. The laboratory-scale CIGS cell efficiency recently reached $20.8 \%$, exceeding the cell efficiency of a multi-crystalline silicon solar cell ${ }^{1,2}$. One of the advantages of the CIGS thin film solar module is the availability of monolithic series interconnections, as illustrated in Figure $1(\mathrm{a})^{3}$. The series connections enable the generation of a higher voltage power from the solar modules (shown in Fig. 1(b)), which can reduce heat loss during electric power transmission. The monolithic interconnections allow adjacent CIGS solar modules to be connected directly in series instead of fabricating, tabbing, and stringing the cells together to make a series of interconnections, as required in silicon wafer solar cells $s^{3}$.

CIGS solar cells were fabricated by depositing a Mo back-contact onto soda-lime glass (SLG), followed by a CIGS absorber layer, a CdS emitting buffer layer, a highly resistive intrinsic $\mathrm{ZnO}$ (i-ZnO) layer, and a transparent conducting oxide (TCO) layer, in sequence. Al-doped $\mathrm{ZnO}$ (AZO) is mainly used as a TCO in CIGS solar cells. The monolithic interconnections in Fig. 1(a) were prepared via three patterning steps between the thin film deposition steps to connect adjacent cells in series ${ }^{3,4}$. The module fabrication sequence was as follows. A Mo backcontact was deposited onto the SLG substrate and scribed using laser ablation along identically spaced patterns (P1 scribing). CIGS/CdS/i-ZnO was sequentially deposited onto the Mo back-contact and mechanically scribed (P2 scribing) using the spacing used during P1 scribing. Finally, the TCO front window layer was deposited on top of the device and mechanically scribed (P3 scribing).

Large-scale modules tend to display a lower power conversion efficiency (PCE) compared to laboratory-scale small cells, because the longer current paths extended over the larger area may limit the ability to reduce electrical resistance besides large-area non-uniformities in the properties of the materials. For instance, thicker front window layer is required for achieving lower series resistance $\left(R_{S}\right)$, but increases light absorption loss within the window layer. Reducing the series resistance without sacrificing the photocurrent is crucial to fabricating large-scale modules. The series resistance consists of the window layer (TCO) resistance, the back contact (Mo) resistance, the absorber (CIGS) resistance, and the non-ohmic junction resistances, such as the Mo/CIGS and CdS/CIGS; however, monolithic integrated modules (as shown in Fig. 1(a)) include additional contacts within the $\mathrm{P} 2$ scribed area consisting of TCO/Mo, which can degrade the performance of the module to a greater or lesser 
(a)

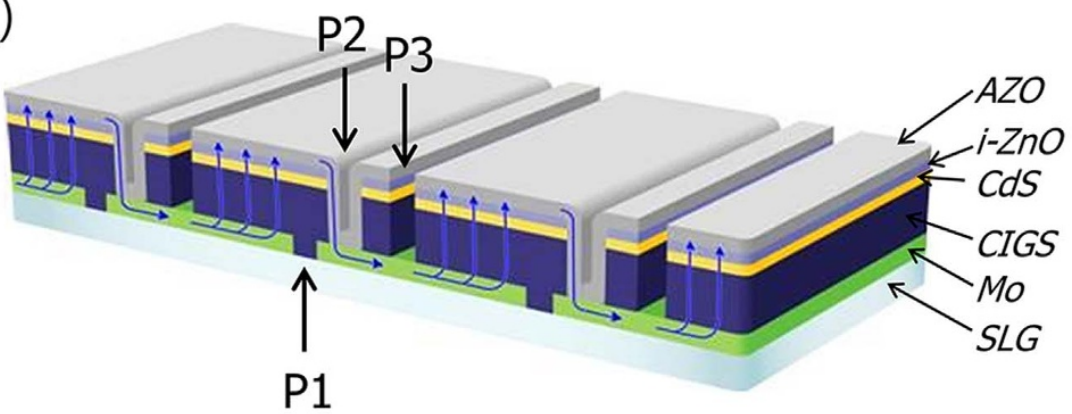

(b)

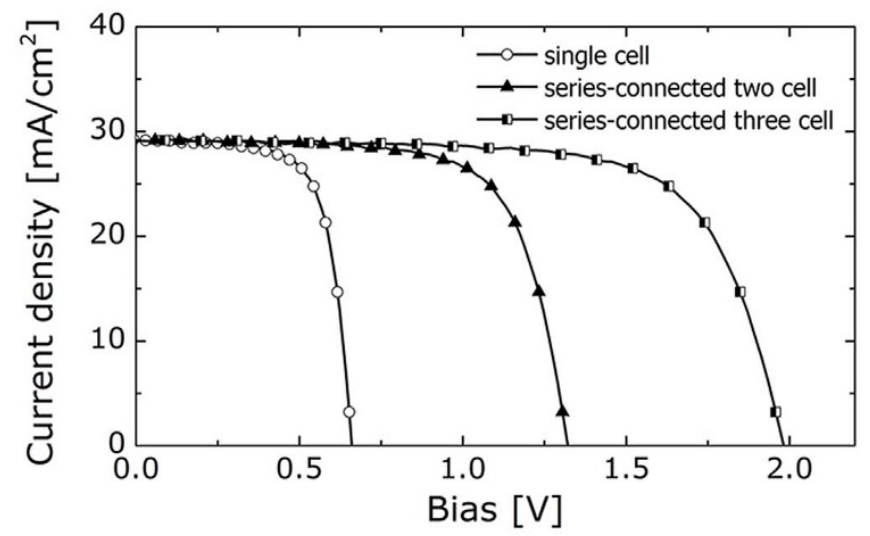

Figure 1 Schematic illustrations of (a) a monolithically integrated CIGS solar module, indicating the photocurrent path, and (b) exemplary highvoltage power generation via a series connection.

degree. It has been challenging to determine whether the $\mathrm{P} 2$ region truly limits the achievement of high-efficiency modules. Unfortunately, the $\mathrm{P} 2$ contact resistance is very difficult to measure using simple methods.

Few methods for measuring the $\mathrm{P} 2$ contact resistance are available. A specially-designed test structure has been suggested using a conventional transmission line method (TLM) concept to measure the TCO sheet resistance and P2 contact resistance ${ }^{5}$. Mo electrodes of a constant width (Mol) were introduced to form contacts with the TCO and guarantee a constant electrode resistance. A Mo electrode with an increasing width (Mo2) was positioned between the pair of Mol fingers to secure the length of the lateral current flow along the TCO. This method required the use of a designed Mo electrode not available in real CIGS modules. In most real monolithically integrated modules, all Mo electrodes have uniform dimensions and are positioned with equal spacing. Thus, the resistances of the solar module are difficult to measure.

This work proposed the use of a simple method for evaluating the P2 contact resistance to avoid special sample fabrication steps and to utilize the existing module structure. The method described here corrected several parasitic components to obtain the exact resistance. These components were not addressed in previous reports. We varied the geometric dimensions of the test structure but tried to reduce the differences between the resistance values measured along different dimensions, and we discussed the parasitic errors of the proposed method in an attempt to calculate the true contact resistance. We also explored the origin of the $\mathrm{P} 2$ contact resistance.

\section{Results and discussion}

P2 contact resistance measurements in the CIGS modules (MTLM). The metal-semiconductor contact resistance can, in general, be measured using a transmission line structure formed from an array of metal contacts having various spacings on top of a single long rectangular semiconductor strip ${ }^{6,7}$. In such structures, the resistance between each pair of metal contacts is the sum of the resistances of two metal-semiconductor contacts and one semiconductor sheet resistance. Thus, the contact resistance and the sheet resistance of a semiconductor may be extracted from a plot of the resistance as a function of the metal contact spacings.

Figure 2(a) illustrates the test structure modified from the CIGS solar modules. P1 and P2 scribing were applied to the CIGS modules, but P3 scribing was not. Then CIGS/CdS/i-ZnO/TCO was shaped into a rectangular stripe of width $\mathrm{W}$ in the middle of the sample. Structures very similar to the transmission line structure could be found in the module, except that the metal contacts (here Mo) were formed prior to the deposition of the semiconductor (here TCO). Application of an electric bias to each pair of Mo contacts permitted the electric current to flow through a $\mathrm{P} 2$ region (TCO/Mo), laterally along the TCO stripe, and again through a P2 region. This current flow path is identical to that in conventional TLMs (see Figs. 2(b) and 2(c)). Note that the current flow between the $\mathrm{P} 2$ contacts was limited laterally along the TCO because the pn junction between the TCO and CIGS isolated the TCO from the highly conductive bottom Mo electrode. Therefore, as illustrated in Fig. $2(\mathrm{~d})$, the total resistance $\left(\mathrm{R}_{\mathrm{TOT}}\right)$ between the zeroth electrode and the i-th electrode could be obtained in the form of equation (1) as a function of the spacings $\left(\mathrm{d}_{\mathrm{i}}\right)$ of the Mo electrode pairs, where the intercept provides the $\mathrm{P} 2$ contact resistance $\left(\mathrm{R}_{\mathrm{C}, \mathrm{P} 2}\right)$ and the slope provides the sheet resistance of TCO $\left(\mathrm{R}_{\mathrm{SH}}\right)$. The advantage of the proposed method is that it allows us to separately measure the $\mathrm{P} 2$ contact resistance $\left(\mathrm{R}_{\mathrm{C}, \mathrm{P} 2}\right)$ and the TCO sheet resistance $\left(\mathrm{R}_{\mathrm{SH}}\right)$ of the real CIGS module. The method can utilize the structure as it is of the monolithically integrated modules prior to P3 scribing step for test structure and, in principle, does not require more work to make special test structures other than the module. In other words, the test structure for $\mathrm{R}_{\mathrm{C}, \mathrm{P} 2}$ and $\mathrm{R}_{\mathrm{SH}}$ is formed on the real solar modules. Hereafter, this method will be referred to as an in-module TLM (M-TLM). 
(a)

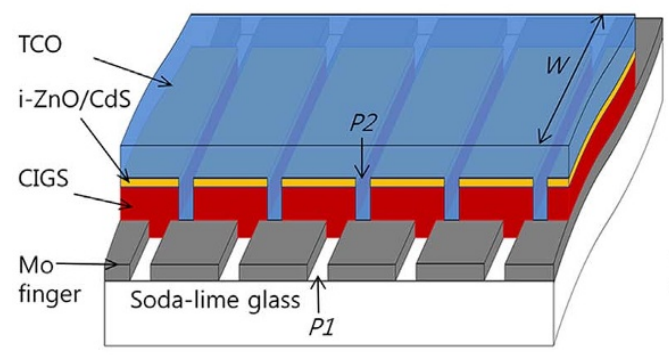

(b)

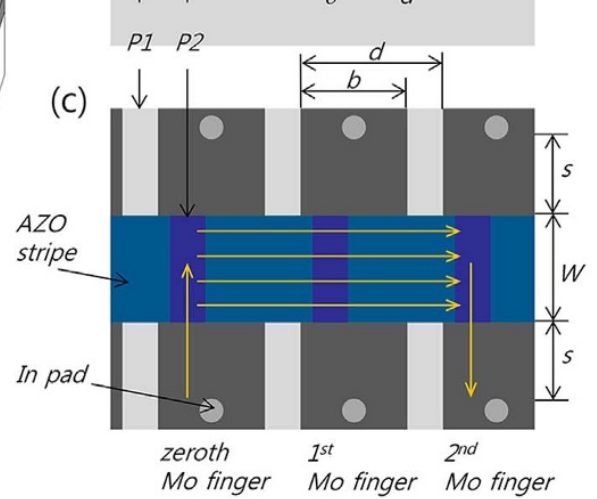

(d)

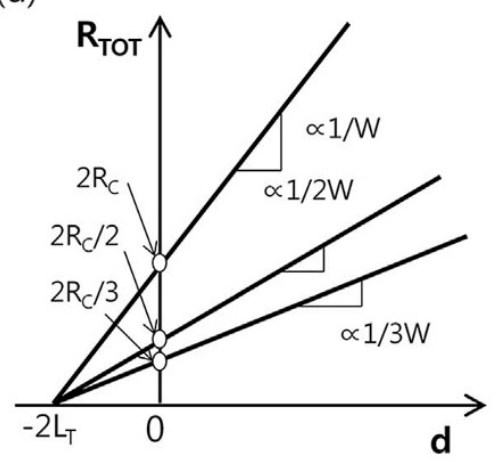

Figure $2 \mid$ The structure used for the in-module transmission line method (M-TLM), modified from the CIGS modules. (a) Three-dimensional view, (b) cross-sectional view, and (c) planar-view; and (d) predicted values of the $\mathrm{R}_{\mathrm{TOT}}$ vs. distance between electrodes for different TCO widths. The yellow arrows represent the current path under a bias applied between the zeroth Mo finger and the $2^{\text {nd }}$ Mo finger.

$$
\left(\mathrm{R}_{\mathrm{TOT}}-\mathrm{R}_{\mathrm{P}}\right)_{\mathrm{i}}=\mathrm{R}_{\mathrm{SH}} \frac{\mathrm{d}_{\mathrm{i}}}{\mathrm{W}}+2 \mathrm{R}_{\mathrm{C}, \mathrm{P} 2},
$$

where W is the width of the TCO rectangular stripe (Fig. 2), $d_{i}$ is the interval between the Mo electrode pairs, which is equal to $i x$ $\mathrm{d}$, where $\mathrm{d}$ is the interval between adjacent $\mathrm{P} 2$ contacts, i.e., the cell-to-cell distance, and $R_{P}$ is the resistance between the probe contact and the metal, forming an external electric connection. In fact, unlike conventional transmission line structures, the resistance between each pair of metals (as represented by $\mathrm{R}_{\mathrm{TOT}}$ in eq. (1)) included contributions from erroneous sources in addition to the above-mentioned $\mathrm{R}_{\mathrm{p}}$. One such contribution was the metal electrode resistance, because the length of the metal fingers across which carriers travelled (to reach the probes) was fairly long, as shown in Fig. 2. This contribution will be discussed later. The other contribution is the uncertainty of the TCO sheet resistance derived from the contribution of the P2 region to the lateral conduction along TCO layer, as elaborated below. Whereas conventional transmission line structures feature a metal electrode array with different interval spacings, this M-TLM structure, which employed the scribed module structure, included equally spaced metal electrodes. More than a P2 region was present between the zeroth electrode and the i-th electrode ( $>$ 2), as shown in Figs. 2(b) and 2(c); the number of $\mathrm{P} 2$ regions that participated in the carrier transport through TCO increased as the distance between each electrode pair increased. Because the Mo electrodes beneath the TCO in the P2 region provided additional paths for carrier transport, the TCO sheet resistance measured in the M-TLM could be erroneously reduced; however, because the width of the P2 region $(50 \mu \mathrm{m})$ was very small compared with the interval between each cell $(\mathrm{d}=5 \mathrm{~mm})$, its contribution to the measured $\mathrm{R}_{\mathrm{SH}}$ was negligible. In particular, the more resistive TCO/Mo interface would suppress the current flow toward the Mo layer out of the TCO layer.

Figure 2(d) illustrates the dependence of $R_{C, P 2}$ and $R_{S H}$ on the width of a TCO rectangular stripe. A larger width resulted in a larger cross-sectional area for carrier transport through the P2 contact region as well as along the TCO, thereby reducing both the TCO sheet resistance $\mathrm{R}_{\mathrm{SH}}$ and the $\mathrm{P} 2$ contact resistance $\mathrm{R}_{\mathrm{C}, \mathrm{P} 2}$, because electrical resistivities are independent of the geometrical dimensions. Here, the contact resistivity could be calculated if the contact area could be accurately defined. In the case of a planar configuration, as in the TLM, the current flow through the interface was crowded around the edge of the metal electrode and reduced away from the edge. Eventually, the current density flowing through the interface was not uniform, thus, a definition of the contact area required the concept of the effective length (referred to as the transfer length) over which most of the current was transferred from the semiconductor into the metal or from the metal into the semiconductor. The transfer length $L_{\mathrm{T}}$ (obtained from the x-intercept of $\mathrm{R}_{\mathrm{TOT}}$ vs. $\mathrm{d}$ in Fig. 2(d)) permitted us to define the specific resistivity, $\rho_{\mathrm{C}, \mathrm{P} 2}$ as follows:

$$
\mathrm{R}_{\mathrm{C}, \mathrm{P} 2}=\frac{\rho_{\mathrm{C}, \mathrm{P} 2}}{\mathrm{~L}_{\mathrm{T}} \mathrm{W}}
$$

for $\mathrm{L}_{\mathrm{T}} \ll \delta$, where $\delta$ is the line width of the P2-scribed region. Otherwise, $\mathrm{L}_{\mathrm{T}}$ should be replaced by $\delta^{7}$.

Evaluations of the P2 contact resistance and the TCO sheet resistance in the CIGS solar module. The M-TLM sample fulfilled its purpose if the resistances measured between adjacent Mo electrodes were sufficiently uniform to provide good linearity in a plot of $R_{\text {Tот }}$ vs. the spacing $\left(d_{i}\right)$ of each Mo electrode pair. Several factors could degrade the resistance uniformity in the M-TLM structure, including non-uniform P1/P2 scribing, or fluctuations in the contact area between the Mo and indium (In) pads due to mechanical pressing. The current-voltage $(I-V)$ characteristics and the resistances between adjacent Mo electrodes were measured over the whole area of the M-TLM samples, as shown in Figs. 3(a) and 3(b). The M-TLM structures provided sufficiently uniform resistances across each Mo pair over the whole area of the submodule (reaching a maximum below a standard deviation of 3\%). The errors increased slightly as the AZO width decreased, possibly due to the enhanced likelihood of a non-uniform AZO stripe width due to the manual mechanical removal of some of the width. As shown in Figs. 3(c) and 3(d), the resistances across each Mo electrode pair increased linearly as the spacing $\left(\mathrm{d}_{\mathrm{i}}\right)$ increased to provide a slope and intercept. Here, the resistance was corrected with the parasitic resistance related to the external connections $\left(R_{p}\right)$.

It is interesting that the $\mathrm{R}_{\mathrm{TOT}}$ values between adjacent electrodes in Fig. 3(b) were not quite different between the AZO widths of $2 \mathrm{~cm}$ and $3 \mathrm{~cm}$. It can be explained by two perspectives. First, besides the AZO resistance, $R_{\text {TOT }}$ included several parasitic resistances such as the external contacts $\left(\mathrm{R}_{\mathrm{p}}\right)$, the Mo/In pad contacts $\left(\mathrm{R}_{\mathrm{C}, \mathrm{Pad} / \mathrm{Mo}}\right)$, and the Mo resistance $\left(\mathrm{R}_{\mathrm{Mo}}\right)$. Since $\mathrm{R}_{\mathrm{p}}$ and $\mathrm{R}_{\mathrm{C}, \mathrm{Pad} / \mathrm{Mo}}$ are fairly large independent of the AZO width, but $\mathrm{R}_{\mathrm{Mo}}$ has a reverse AZO-width dependency to the AZO resistance, the difference of $R_{T O T}$ between $2 \mathrm{~cm}$ and $3 \mathrm{~cm}$ was reduced as compared with that predicted by the AZO resistance. Second, as shown in Fig. 2(c), the current flows 

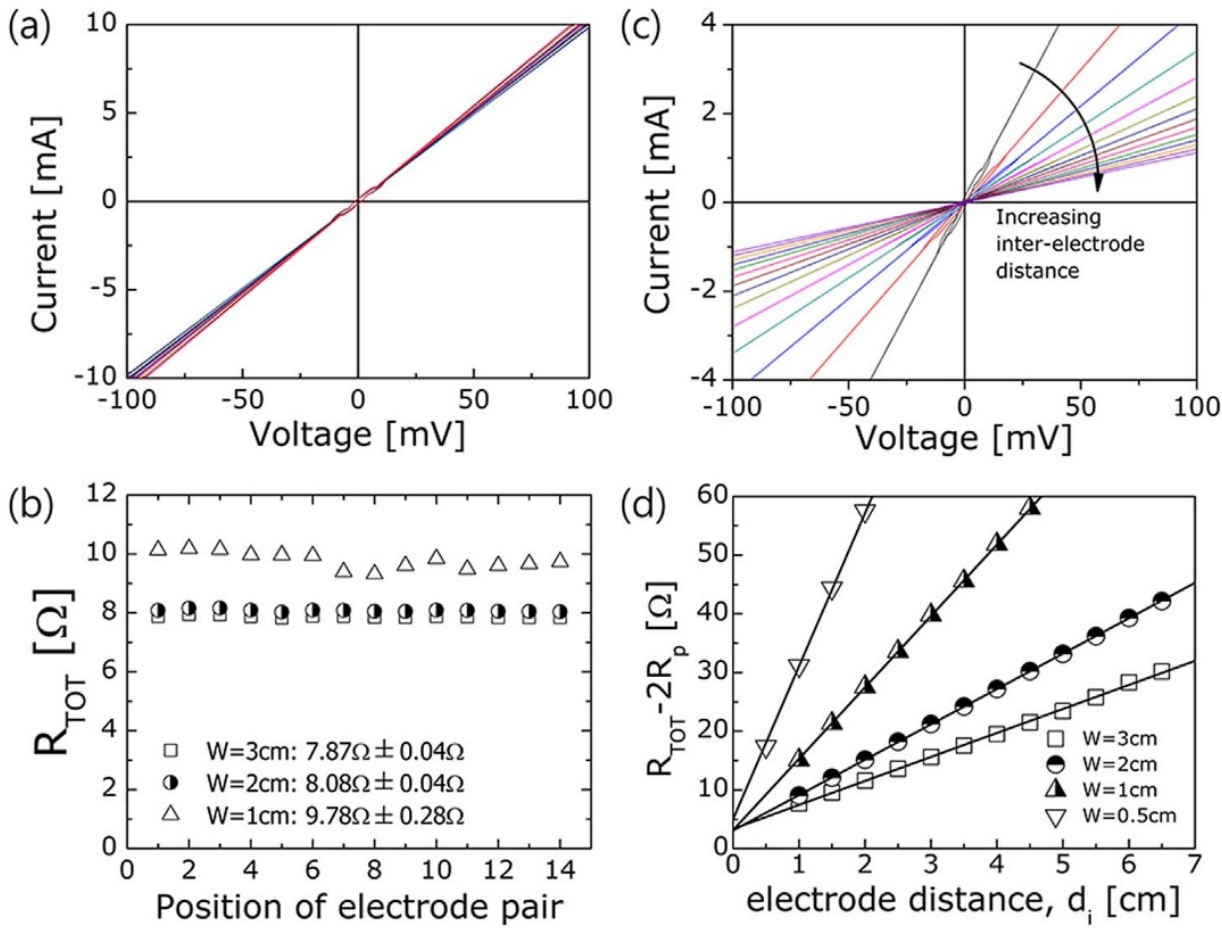

Figure $3 \mid$ (a) $I$ - $V$ characteristics obtained between adjacent electrode pairs in the M-TLM sample, i.e., for a $1 \mathrm{~cm}$ wide AZO rectangular stripe. (b) Resistance distributions obtained from the various AZO widths, and (c) $I$ - $V$ characteristics as a function of the distance between electrode pairs, i.e., for a $1 \mathrm{~cm}$ wide $\mathrm{AZO}$ rectangular stripe, and $(\mathrm{d})$ the resistance vs. electrode interval $\left(\mathrm{d}_{\mathrm{i}}\right)$ as a function of the AZO width.

along a Mo finger from probe contact (In pad) and changes its direction to perpendicular when it enters the AZO stripe through P2 contact. Such a change of the current direction would induce the current crowding at P2 contact around the edge of AZO stripe facing the In pad, and subsequently induce the current spreading out wider AZO stripe. Since the portion of AZO stripe between adjacent Mo electrodes (with an interval of $5 \mathrm{~mm}$ ) is very small as compared of the AZO width of $2 \mathrm{~cm}$ or $3 \mathrm{~cm}$, it mostly experiences the current spreading. Thus, the effective dimension of the AZO width contributing to current flow would be reduced from an apparent value $\mathrm{W}$ to smaller, and its reduction degree would increase as the AZO width increases. The reduction of the AZO resistance with increasing AZO width would be mitigated in the case of adjacent Mo electrodes than predicted. We believe that two points elaborated above are the reason why the $\mathrm{R}_{\mathrm{TOT}}$ of $2 \mathrm{~cm}$ and $3 \mathrm{~cm}$ are similar. In accordance with the speculation, the resistance between adjacent electrodes tended to deviate from the linearity of $\mathrm{R}_{\text {TOт }}$ vs. $\mathrm{d}$ in the case of relatively large $A Z O$ width, and thus the data was not used in the plot of $R_{T O T}-2 R_{p}$ vs. d, as shown in Fig. 3(d).

Figure 3(d) shows that different AZO widths (W) produced different slopes and intercepts, as predicted in the previous section. Figure 4(a) shows that the AZO sheet resistance $\left(\mathrm{R}_{\mathrm{SH}}\right)$ could be calculated from the slope, and the apparent P2 contact resistance (intercept/2) was obtained from the intercept as a function of the AZO width. Because $R_{S H}$ and $\rho_{\mathrm{C}, \mathrm{P} 2}$ were independent of the AZO width, the slope and intercept depended inversely on the AZO width, as illustrated in Fig. 2(b). The slope in Fig. 3(d) agreed well with the expected dependence on the AZO width, yielding nearly identical $\mathrm{R}_{\mathrm{SH}}$ values in the range of $12.1-12.5 \Omega$, regardless of the AZO width. Consistent with the measured data, the sheet resistances of the AZO films formed on the SLG using an LG Innotek pilot process were 12.0 $\pm 0.5 \Omega$, as measured independently by the four-point probe method. These results indicated that the M-TLM test accurately measured the TCO resistance in the CIGS solar module, which was otherwise nearly impossible to evaluate.
The intercept (from which the contact resistance could be estimated) exhibited an AZO width dependence that differed from expectations in that it increased with decreasing width. The intercept remained constant as the AZO width decreased from $3 \mathrm{~cm}$ to $1 \mathrm{~cm}$ and increased significantly below $1 \mathrm{~cm}$. The deviations of the intercept from the geometry dependence suggested the presence of measurement error sources in the test. Unless the errors could be corrected, the suggested M-TLM test would not be useful. Various possible error sources will be discussed further, below.

The M-TLM structure, as shown in Fig. 2(a), indicated that the current flowed from one probe to the other through the In pad, through the In/Mo interface, along the Mo electrode fingers laterally along the scribing direction, through the $\mathrm{P} 2$ contact (Mo/AZO interface), then along the AZO layer laterally transverse to the scribing lines, through the $\mathrm{P} 2$ contact, along the Mo layer, through the $\mathrm{In} / \mathrm{Mo}$ interface, and through the In pad, as illustrated in Figs. 2(b) and 2(c). Two parasitic resistances $\left(\mathrm{R}_{\mathrm{PS}}\right)$, including the Mo resistance and the pad-to-Mo contact resistance, affected the resistance measurements between each Mo electrode pair. Equation (1) could be modified according to:

$$
\begin{aligned}
\mathrm{R}_{\mathrm{TOT}}-2 \mathrm{R}_{\mathrm{P}} & =\mathrm{R}_{\mathrm{SH}} \frac{\mathrm{d}_{\mathrm{i}}}{\mathrm{W}}+2 \mathrm{R}_{\mathrm{C}, \mathrm{P} 2}+\mathrm{R}_{\mathrm{PS}} \\
& =\mathrm{R}_{\mathrm{SH}} \frac{\mathrm{d}_{\mathrm{i}}}{\mathrm{W}}+2 \mathrm{R}_{\mathrm{C}, \mathrm{P} 2}+2 \mathrm{R}_{\mathrm{Mo}}+2 \mathrm{R}_{\mathrm{C}, \mathrm{pad} / \mathrm{Mo}}
\end{aligned}
$$

where $R_{M o}$ is the resistance of the Mo electrode along the scribing direction, which depended on the Mo dimensions, and $\mathrm{R}_{\mathrm{C}, \mathrm{pad} / \mathrm{Mo}}$ is the In/Mo contact resistance, which was independent of the geometry. Assuming that the distribution of current in the Mo and AZO layers was uniform along the direction transverse to the respective current flows (which were parallel to the scribing line in the Mo but transverse to the scribing line in AZO), $\mathrm{R}_{\mathrm{Mo}}$ could be expressed in the form of $\mathrm{R}_{\mathrm{SH}, \mathrm{Mo}}(\mathrm{s}+\mathrm{W} / 2) / \mathrm{b}$, where $\mathrm{R}_{\mathrm{SH}, \mathrm{Mo}}$ is the sheet resistance of the Mo electrode, $s$ is the length from the In pad to 
(a)

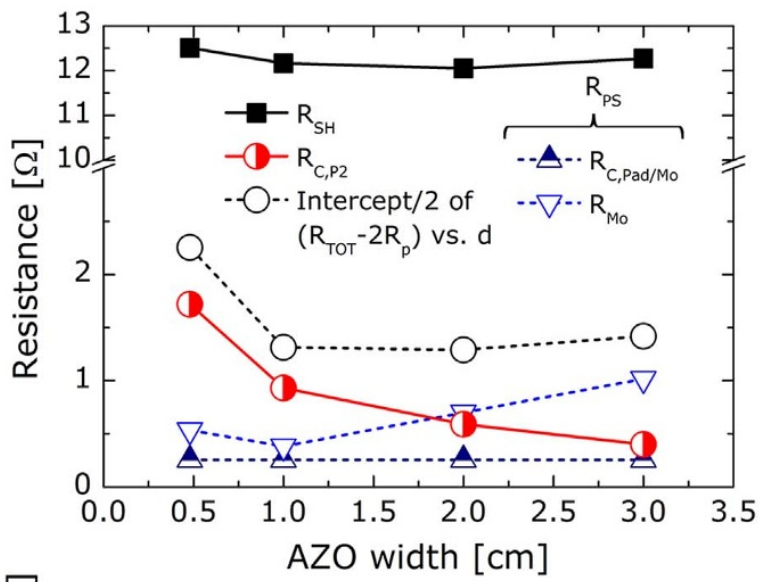

(b)

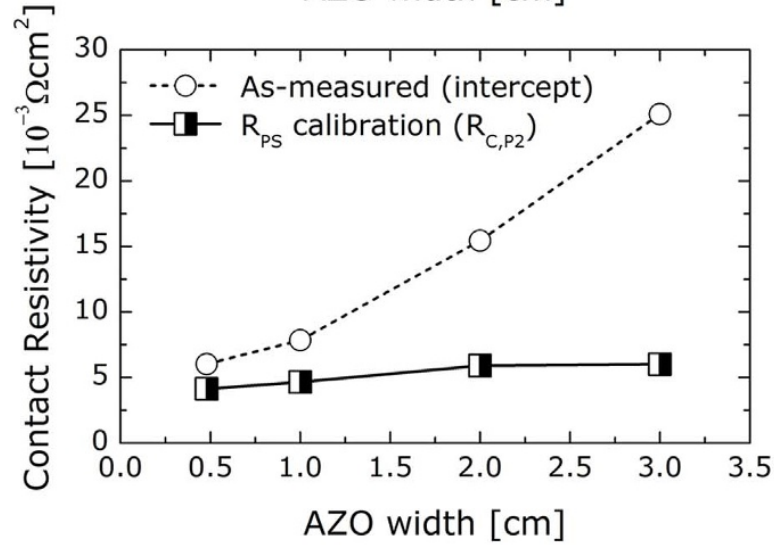

Figure $4 \mid$ The AZO width dependence of (a) the measured resistances $\left(\mathrm{R}_{\mathrm{SH}}, \mathrm{R}_{\mathrm{C}, \mathrm{P} 2}, \mathrm{R}_{\mathrm{PS}}\right.$ ) and (b) the $\mathrm{P} 2$ contact resistivity, as calculated from the $\mathrm{P} 2$ contact resistances presented in (a).

the closer edge of the AZO rectangular stripe, and $\mathrm{b}$ is the width of each Mo finger ( $\cong \mathrm{d}$, see Fig. $2(\mathrm{~b})$ ). $\mathrm{R}_{\mathrm{C} \text {,pad/Mo was experimentally }}$ measured through application of the TLM concept. The similar size and shape of In pad array was formed on an exposed Mo finger with increasing spacings between adjacent In pads and then the resistances between each In pair was measured. $\mathrm{R}_{\mathrm{C}, \mathrm{pad} / \mathrm{Mo}}$ was obtained from the intercept in the plot of resistance vs. electrode interval.

The parasitic resistances $\left(\mathrm{R}_{\mathrm{PS}}\right)$ of $\mathrm{R}_{\mathrm{Mo}}$ and $\mathrm{R}_{\mathrm{C} \text {,pad/Mo }}$ were obtained according to the procedures described above, as summarized in Fig. 4(a), as a function of the width of the AZO stripe. The contact resistance, including $\mathrm{R}_{\mathrm{C} \text {,pad/Mo }}$, remained constant because the $\mathrm{In} /$ Mo contact remained unchanged as the AZO width was varied. As the AZO width decreased from $3 \mathrm{~cm}$ to $1 \mathrm{~cm}$, the effective length of the current flow along the Mo fingers (defined as $\mathrm{s}+\mathrm{W} / 2$ ) decreased because s was fixed and $\mathrm{W}$ was reduced, as mentioned in the Methods section, leading to a decrease in $\mathrm{R}_{\mathrm{Mo}}$. Below $1 \mathrm{~cm}$, however, the distance (s) from the In pad to the facing edge of the AZO stripe increased because the facing edge of the stripe was mechanically removed to reduce the width, as mentioned in the Methods section. Variations in $\mathrm{R}_{\mathrm{Mo}}$ accounted for the constant $\mathrm{P} 2$ contact resistance (extracted directly from the intercept) for AZO widths of $1-3 \mathrm{~cm}$. A reduction in $\mathrm{R}_{\mathrm{Mo}}$ with the decreasing $\mathrm{AZO}$ width may have alleviated the increase in the P2 contact resistance expected from Fig. 2(d). $\mathrm{R}_{\mathrm{C}, \mathrm{P} 2}$, calculated based on the intercept value and the two parasitic resistances, more closely followed the values expected from the AZO width dependency, as shown in Fig. 4(a). This result was apparent in a plot of the contact resistivity as a function of the AZO width, as shown in Fig. 4(b). The contact resistivity was calculated according to eq. (2), in which $\mathrm{L}_{\mathrm{T}}$ was replaced by the P2 scribing line width ( $\delta$ : $50 \mu \mathrm{m}$ ) because the $\mathrm{L}_{\mathrm{T}}$ value calculated according to the definition in Fig. 2(d) was much higher than $\delta$. The contact resistivity is a material property that is independent of geometry factors, such as the AZO width. Figure 4(b) shows that in the M-TLM test, the value of $R_{P S}$ was successfully used to calculate the real $\mathrm{P} 2$ contact resistivity $\rho_{\mathrm{C}, \mathrm{P} 2}$, which was independent of the AZO width.

The procedure described above successfully demonstrated that the M-TLM test could be used to evaluate the AZO sheet resistance and the P2 contact resistance in a CIGS monolithically integrated module. The effects of the P2 contact resistance on PCE were explored by calculating the contribution to the series resistance $\left(R_{S}\right)$. The contribution of $\mathrm{R}_{\mathrm{C}, \mathrm{P} 2}$ to the series resistance (per unit area) could be calculated according to $\mathrm{R}_{\mathrm{S}}=\rho_{\mathrm{C}, \mathrm{P} 2} \times \mathrm{b} / \delta$ (here $\mathrm{b} \cong 0.5 \mathrm{~cm}$ and $\delta=$ $0.005 \mathrm{~cm}$ ), using the measured contact resistivities, ranging from $0.4 \Omega \mathrm{cm}^{2}$ at an AZO width of $0.5 \mathrm{~cm}$ to $0.6 \Omega \mathrm{cm}^{2}$ at an AZO width of $3 \mathrm{~cm}$. For comparison, the contributions of the AZO electrode to the series resistance were calculated using the measured $\mathrm{R}_{\mathrm{SH}}$ values shown in Fig. 4(a), which were found to be $0.38 \Omega \mathrm{cm}^{2}$ (this value was calculated as described in the Methods section). The series resistance for each cell was independently calculated from the light current density-voltage $(J-V)$ characteristics, and was found to be $0.81 \Omega \mathrm{cm}^{2}$, on average ${ }^{8}$. The sum of the series resistances due to the P2 contact and the TCO agreed well with the total series resistance. This result suggested that the M-TLM test provided fairly reliable values for the $\mathrm{P} 2$ contact resistance, although the $\mathrm{P} 2$ contact resistance was likely be slightly erroneous.

The error level was potentially $20 \%$, considering the range of measured values, as mentioned above. In general, the errors, related to the AZO width, could be attributed to the area inhomogeneity in P2 contact properties. Besides, there could be any errors in calibrating the measured data to get the contact resistivity of Fig. 4(b). The current flow was not along a single direction in the M-TLM structure unlike the conventional TLM. As seen in Fig. 2(c), it flowed largely along the scribing direction in the Mo electrode line, but would change its direction into perpendicular when it entered AZO layer through the P2 contact. As stated above, the change in the current direction would induce the current crowding at the P2 contact around the edge of the AZO stripe facing the external electrical connection (the In pad). Subsequently, the current from the crowded P2 contact region would be spread out over whole cross-section of the AZO stripe. The hypothesis would, to some degree, break the assumptions of our method; the uniform current distribution in the Mo fingers and the AZO stripe, as stated before. The current crowding effect would reduce the effective lengths of the Mo finger beneath the AZO stripe and of the $\mathrm{P} 2$ contact region participating truly in the carrier transport. The effect would influence the calculation accuracies of $\mathrm{R}_{\mathrm{Mo}}$ and the contact resistivity $\left(\rho_{\mathrm{C}, \mathrm{P} 2}\right)$. In addition, the accuracy would depend on the geometric dimension such as the AZO width, because the gap between the real current flow and our simple assumption would increase with increasing width. For the reason, although the exact numbers cannot be given here, we can say qualitatively that the gradual increase of contact resistivity with the AZO width should also be attributed to the geometric dependency of the calculation errors. More accurate calculations of the current flow based on a three-dimensional finite element analysis (FEA) may provide a higher degree of accuracy to the suggested M-TLM test.

Finally, the P2 contact resistance influenced the series resistance in a manner comparable to the TCO resistance, substantially contributing to the degradation of the fill factor of the solar modules. Therefore, the achievement of higher-efficiency solar modules requires appropriate improvements to the $\mathrm{P} 2$ contact region based on understanding of the origin of the $\mathrm{P} 2$ contact resistance.

The origin of the $\mathrm{P} 2$ contact resistance. The $\mathrm{P} 2$ contact resistance $\left(\mathrm{R}_{\mathrm{C}, \mathrm{P} 2}\right)$ may have originated from either the AZO/Mo contact itself or from the $\mathrm{MoSe}_{2}$ phase formed on the Mo surface during CIGS deposition. These two hypotheses were tested by comparing the 


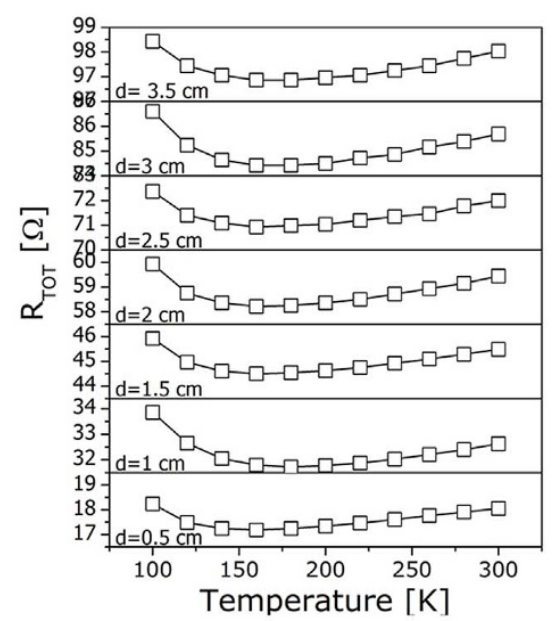

(a)

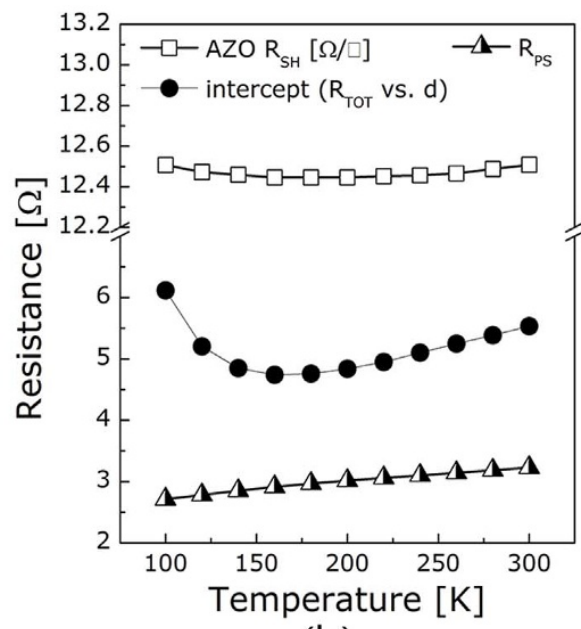

(b)

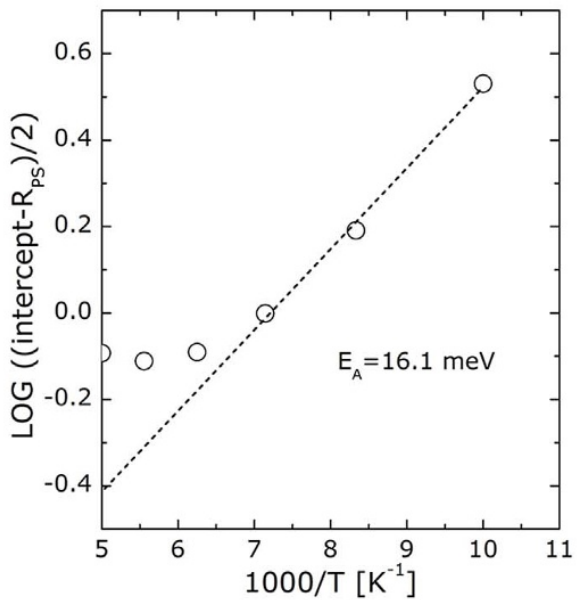

(c)

Figure $5 \mid$ (a) $\mathrm{R}_{\mathrm{TOT}}$ as a function of temperature, for various electrode pair distances. (b) The $\mathrm{R}_{\mathrm{SH}}$ and intercept extracted from the M-TLM test as a function of the temperature, together with the electrode-related parasitic resistance, as measured from a single reference Mo finger. (c) The activation energy for carrier transport related to the $\mathrm{P} 2$ contact. An AZO sample width of $0.5 \mathrm{~cm}$ was used for this measurement.

measured activation energies $\left(\mathrm{E}_{\mathrm{A}}\right)$ for carrier transport through the $\mathrm{P} 2$ contacts in $\mathrm{AZO} / \mathrm{Mo}$ and in $\mathrm{AZO} / \mathrm{MoSe}_{2} / \mathrm{Mo}$.

The M-TLM test procedure was applied to a sample with an AZO width of $0.5 \mathrm{~cm}$ at various temperatures to obtain the activation energy. Figure 5(a) shows the temperature dependence of the resistances of each Mo electrode pair having different intervals $(0.5,1,1.5$, $2,2.5,3,3.5 \mathrm{~cm})$. The AZO $\mathrm{R}_{\mathrm{SH}}$ and intercept at various temperatures could be extracted from a plot of $\mathrm{R}_{\mathrm{TOT}}$ vs. the Mo electrode spacings reconstructed from Fig. 5(a), as shown in Fig. 5(b). The values of the AZO $R_{S H}$ did not vary with temperature, but the intercept did vary significantly as the temperature was changed. The derivative of the intercept as a function of the temperature was negative at low temperatures (similar to the behavior of semiconductors) but positive at high temperatures (similar to the behavior of metals). It seemed that the intercept value across the In pad, In/Mo interface, Mo electrode, and P2 contact has mixed contributions from the semiconductor and metal.

In order to more accurately obtain the activation energy related to the P2 contact, the parasitic resistances were independently measured as a function of temperature during the M-TLM test. An additional In pad was attached to each Mo electrode finger opposite to the original pad position across the AZO/CIGS rectangular stripe (see Fig. 2(c)), and the resistance across a pair of In pads on a Mo finger was measured as a function of the temperature. The contributions of the main parasitic resistances generated by the probe-In contact, the In/Mo contact, and the Mo layer, were measured. Figure 5(b) shows that $\mathrm{R}_{\mathrm{PS}}$ increased with temperature, indicating that the parasitic components were largely metallic. The temperature dependence of the intercept at high temperatures was derived mainly from the metallic parasitic components. The real value of the $\mathrm{P} 2$ contact resistance was obtained by subtracting $\mathrm{R}_{\mathrm{PS}}$ from the intercept and then dividing the value by two ((intercept $\left.\left.-\mathrm{R}_{\mathrm{PS}}\right) / 2\right)$. Figure $5(\mathrm{c})$ shows that the fit of the $\mathrm{P} 2$ contact resistance to an Arrhenius-type equation $\left(\mathrm{R}_{\mathrm{C}, \mathrm{P} 2}(\mathrm{~T}) \propto \exp \left(-\mathrm{E}_{\mathrm{A}} / \mathrm{kT}\right)\right)$ resulted in an activation energy of $16.1 \mathrm{meV}$.

The activation energies of the AZO/Mo interfaces prepared in a more controlled manner were measured and compared to explore the nature of the P2 contact. The measurements were conducted using an inverse-type TLM structure (hereafter referred to as iTLM), as illustrated in Fig. 6(a), fabricated such that the Mo electrode array was formed prior to the AZO rectangular strip (see Fig. 6(b) for an optical microscopy image of the structure). The resistivity of the $\mathrm{AZO} / \mathrm{Mo}$ interface was thought to derive mainly from the $\mathrm{MoSe}_{2}$ phase between the AZO and Mo materials. Although the $\mathrm{MoSe}_{2}$ layer produced via a reaction of Mo with Se vapor during the CIGS deposition step may have benefitted the CIGS/Mo junction properties by reducing the junction barrier ${ }^{9,10}$, this layer tends to provide a fairly high electrical resistivity. The effect of this layer was characterized by preparing two AZO/Mo interfaces by depositing AZO films onto fresh or selenized Mo electrode arrays. As shown in the transmission electron microscopy (TEM) image presented in Fig. 6(c), the Mo surface was selenized successfully at $450^{\circ} \mathrm{C}$ to form a very thin $\mathrm{MoSe}_{2}$ planar structure 5-10 nm thick. The TEM observation over wider area showed that the $\mathrm{MoSe}_{2}$ interlayer was formed uniformly

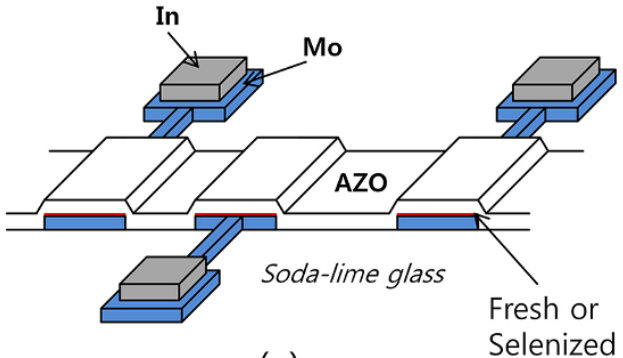

(a)
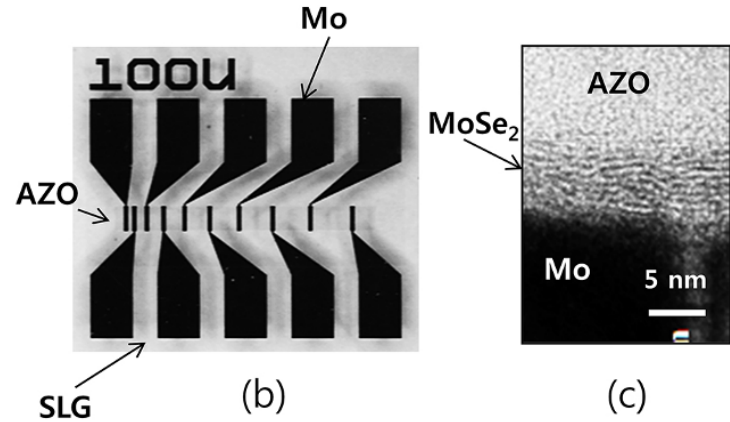

(c)

Figure 6 (a) Illustration of the inverse TLM (i-TLM) sample used to measure the AZO/Mo contact resistance. (b) Optical microscopy image of the asfabricated i-TLM sample, and (c) TEM cross-sectional image of the AZO/selenized Mo. 

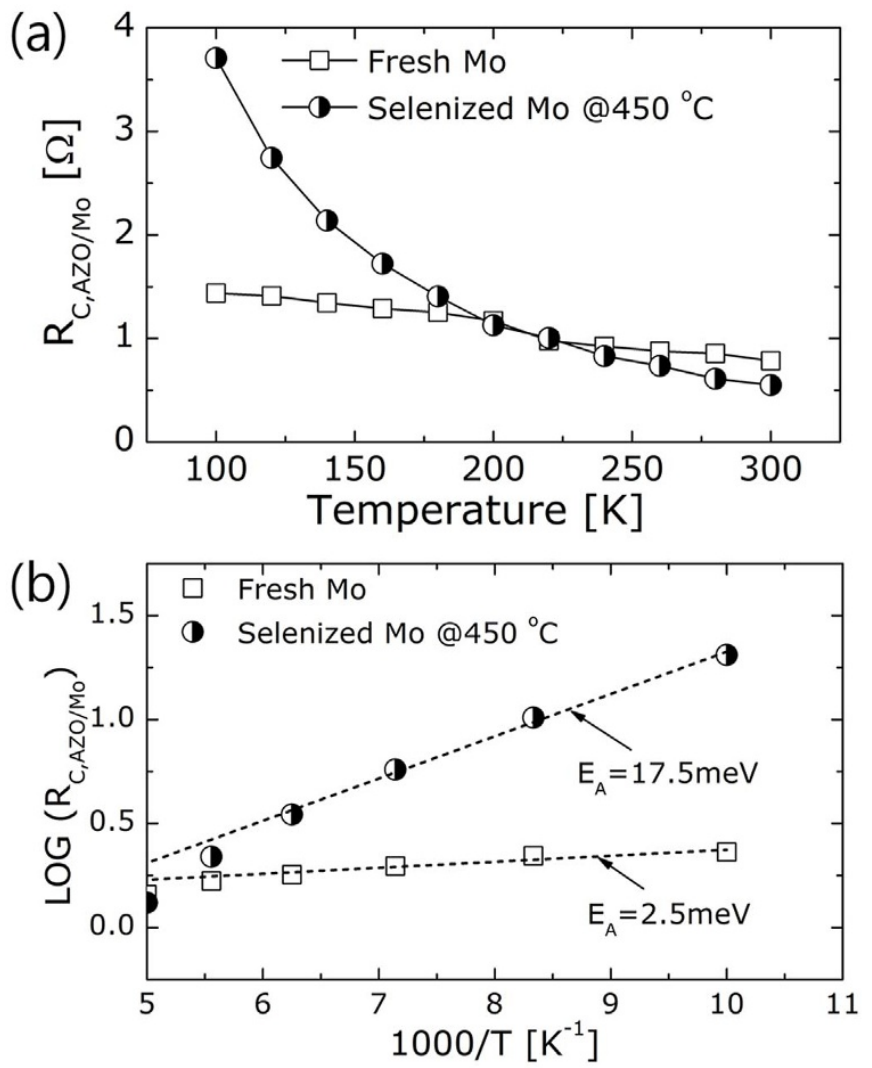

Figure 7 | Comparison of the AZO/selenized Mo with an AZO/fresh Mo. (a) The contact resistance as a function of the temperature, as measured using i-TLM and (b) the activation energies calculated from (a).

over the whole Mo surface. The parasitic resistances were corrected, as in the M-TLM test case (as described above) by measuring the resistance of a reference Mo electrode prepared to have the same length and current flow cross-section as each Mo electrode pair involved in the i-TLM tests. Figure 7(a) compared the $\mathrm{R}_{\mathrm{PS}}$-corrected contact resistances of the $\mathrm{AZO} / \mathrm{Mo}\left(\mathrm{R}_{\mathrm{C}, \mathrm{AZO} / \mathrm{Mo}}\right)$ with the fresh and selenized Mo layers as a function of the temperature. The $\mathrm{R}_{\mathrm{C}, \mathrm{AZO} / \mathrm{Mo}}$ values obtained from the selenized Mo layer displayed a stronger temperature dependence than those obtained from the fresh Mo layer. The activation energies of the contacts were $2.5 \mathrm{meV}$ for the fresh Mo layer and $17.5 \mathrm{meV}$ for the selenized Mo layer, as shown in Fig. 7(b). These results indicated that the barrier to carrier transport was negligible in the contact between the AZO and fresh Mo layers, although it increased substantially upon the selenization of Mo. The activation energy at the $\mathrm{MoSe}_{2}$ interface agreed well with the measurements reported previously using a similar method for the CIGS/ Mo interface ${ }^{11}$, confirming that the $\mathrm{MoSe}_{2}$ layer itself was resistive, regardless of the surrounding material species.

A comparison of Figs. 5(c) and 7(b) revealed that the activation energy ( $16.1 \mathrm{meV}$ ) of the $\mathrm{R}_{\mathrm{C}, \mathrm{P} 2}$ in the module was very similar to that of the $\mathrm{AZO} / \mathrm{MoSe}_{2} / \mathrm{Mo}$ contact resistance $(17.5 \mathrm{meV}) . \mathrm{R}_{\mathrm{C}, \mathrm{P} 2}$ was hypothesized to originate from the presence of a residual $\mathrm{MoSe}_{2}$ layer in the $\mathrm{P} 2$ region, even after mechanical scribing. This hypothesis was tested by imaging the cross-section of the $\mathrm{P} 2$ scribing region using scanning transmission electron microscopy (STEM). Figure 8 showed that the CIGS $\backslash \mathrm{CdS} \backslash \mathrm{i}-\mathrm{ZnO}$ layers were completely removed after P2 mechanical scribing, but the $\mathrm{MoSe}_{2}$ phases between the AZO and Mo layers remained intact. These results provided strong evidence supporting the assertion that residual $\mathrm{MoSe}_{2}$ in the P2 contact region was responsible for $\mathrm{R}_{\mathrm{C}, \mathrm{P} 2}$. Notably, the $\mathrm{P} 2$ contact resistance was significantly enhanced after long exposure times to high-temperature high-humidity environments, such as the damping test conditions described previously ${ }^{5}$. Our results indicated that the degradation of $\mathrm{R}_{\mathrm{C}, \mathrm{P} 2}$ was closely related to the presence of the $\mathrm{MoSe}_{2}$ phases in the $\mathrm{P} 2$ region. In other words, the planar structure of the P2-MoSe $e_{2}$ phase was similar to that of graphite ${ }^{12}$ would be structurally distorted or corrupted in the presence of moisture under harsh conditions.

The complete removal of the $\mathrm{MoSe}_{2}$ layer during the P2 scribing process or the modification of the $\mathrm{MoSe}_{2}$ layer to reduce the resistivity were crucial to reducing the series resistance of the CIGS solar modules and enhancing their long-term stabilities. In fact, the $\mathrm{MoSe}_{2}$ layer with a graphite-like structure altered its atomic orientations and layer thickness, depending on various parameters, including the Mo density, temperature, or presence of $\mathrm{Na}^{9,11,13}$. The resistivity of the layer also depended on its atomic orientations and $\mathrm{Na}$ doping levels ${ }^{11,13}$. Therefore, in an effort to increase the performances of the CIGS solar modules, we could find innovative routes for the

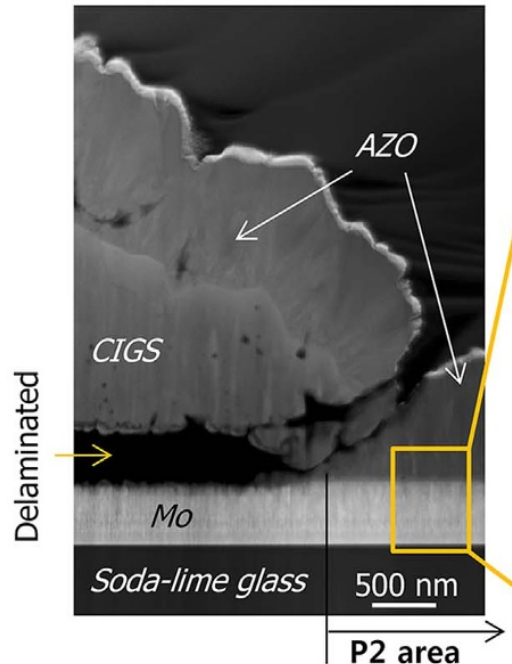

(a)

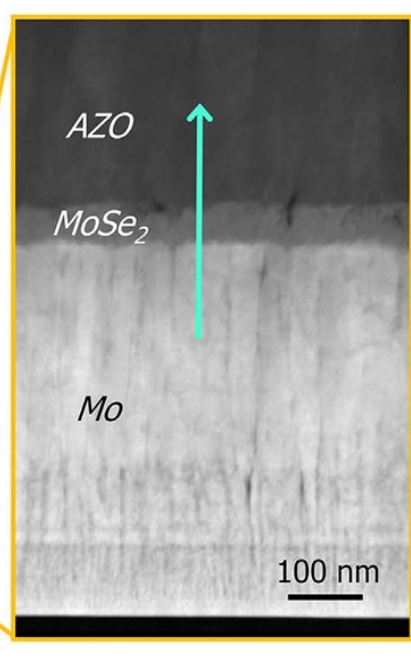

(b)

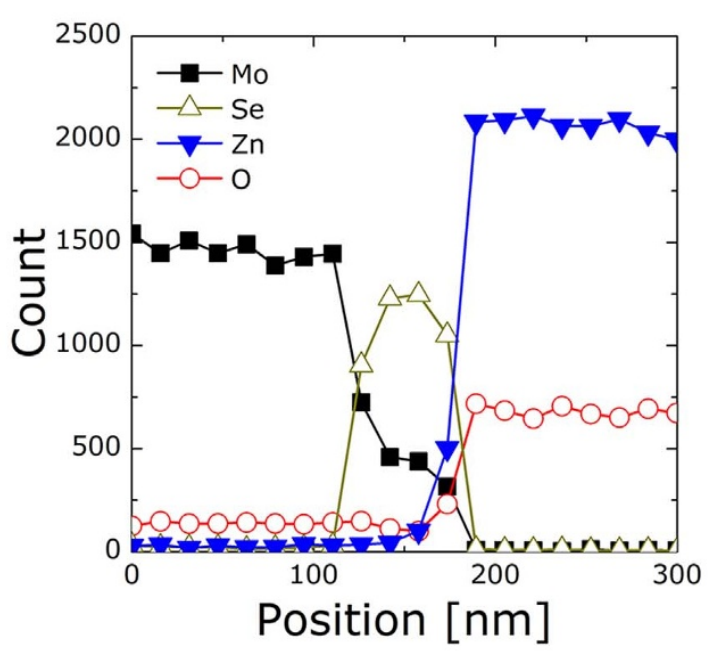

(c)

Figure 8 STEM analysis of the P2 contact region in a monolithically integrated CIGS module. (a) Cross-sectional image, (b) magnified image showing the $\mathrm{MoSe}_{2}$ phase residing between the $\mathrm{AZO}$ and Mo layers in the $\mathrm{P} 2$ region, and (c) its corresponding composition profile along the line indicated in (b). 
modification of $\mathrm{MoSe}_{2}$ structure to reduce the resistivity and increase the stability, in addition to improving the P2 scribing technology to completely remove the $\mathrm{MoSe}_{2}$ layer. This approach may require a deeper understanding of the $\mathrm{MoSe}_{2}$ structure and its relationship to the mechanical and electrical properties of the material.

\section{Summary and conclusions}

In this work, we suggested a simple approach to measuring the contact resistance of the $\mathrm{P} 2$ region and the sheet resistance of a front window layer (AZO). In the absence of this approach, these measurements are very difficult to make in monolithic integrated modules. This study did not rely on specially designed, but rather on commercially-available CIGS solar modules, except that no P3 scribing was applied to isolate the AZO layer. We successfully measured the P2 contact resistance and the sheet resistance of the AZO layer in the CIGS solar module. The contact resistance of the $\mathrm{P} 2$ region was found to increase the series resistance of the solar modules to some degree. This contribution was comparable to that of the AZO window layer, although the accuracy of this measurement may depend on the accuracy of the electrode resistance corrections. Furthermore, the $\mathrm{P} 2$ contact resistance was demonstrated to arise from the $\mathrm{MoSe}_{2}$ phases present between the TCO and Mo layers in the P2 region by comparing the activation energies for carrier transport in the AZO/ Mo and $\mathrm{AZO} / \mathrm{MoSe}_{2} / \mathrm{Mo}$ structures and by examining the TEM cross-sectional images of the $\mathrm{P} 2$ region.

\section{Methods}

Sample preparation for the M-TLM test. The CIGS modules used in this experiment were fabricated using an LG Innotek pilot process line that provided several small devices around $10 \mathrm{~cm}$ in length cut from monolithically interconnected modules 370 $\times 470 \mathrm{~mm}^{2}$ in size. A typical CIGS cell structure (soda-lime glass/Mo/CIGS/CdS/i$\mathrm{ZnO} / \mathrm{AZO}$ ) and typical processes were employed. After the Mo back contact layer was deposited via direct current sputtering, then P1-patterned by a laser scribing system to have $5 \mathrm{~mm}$ interval spacings. The CIGS, CdS, and i-ZnO layers were sequentially deposited via three-stage coevaporation, chemical bath deposition, and DC sputtering, respectively $\mathrm{y}^{14}$. The CIGS/CdS/i-ZnO layers were P2 patterned using a mechanical scribing system to yield $5 \mathrm{~mm}$ spacings. The scribing line width was $60 \mu \mathrm{m}$ for P1 and $50 \mu \mathrm{m}$ for P2. Consistent with the M-TLM principles, P3 scribing was not applied. The M-TLM test structure was prepared by mechanically removing part of the CIGS/CdS/i-ZnO/AZO layer to form a long rectangular stripe $0.5,1,2$, or $3 \mathrm{~cm}$ wide. Each width was obtained by sequentially removing one side edge from the initially formed $3 \mathrm{~cm}$ wide rectangular stripe in a single sample. The samples with rectangular stripes $1-3 \mathrm{~cm}$ in width were prepared by removing the layer from the edge of the stripe opposite to the electric pad, whereas the sample with the stripe $0.5 \mathrm{~cm}$ in width was prepared by removing the layer from the edge (of the stripe $1 \mathrm{~cm}$ in width) facing the electric pad.

Sample preparation for the i-TLM test. The contact resistances of the AZO/Mo structures prepared with a controlled interface were compared by preparing TLM samples with inverse structures on the micro scale (as shown in Figs. 6(a) and (b)). An AZO film with a long stripe was placed on top of an array of patterned Mo electrodes. The fabrication procedure is as follows. The Mo films were wet-etched in a solution containing $30 \mathrm{~mL} \mathrm{H}_{3} \mathrm{PO}_{4}, 18 \mathrm{~mL} \mathrm{HNO}_{3}, 10 \mathrm{~mL} \mathrm{CH}_{3} \mathrm{COOH}$, and $65 \mathrm{~mL}$ deionized water along with a photoresist mask that produced an electrode array with increasing spacings. Long stripes of the AZO films (deposited by RF-sputtering) were then patterned via a lift-off process. The effects of the $\mathrm{MoSe}_{2}$ layer present between the $\mathrm{AZO}$ and Mo layers on the contact were investigated by selenizing some of the Mo samples under high-flux Se at $450^{\circ} \mathrm{C}$ in a co-evaporation chamber prior to $\mathrm{AZO}$ film deposition.

Measurement of resistances in M-TLM and i-TLM. The current-voltage (I-V) characteristics as a function of the interelectrode distance were measured using a Keithley 2400 source measure unit (SMU) connecting each pair of Mo electrodes. The external electrical connection was formed by attaching the Indium pad to the exposed Mo via mechanical pressing to reduce the Mo surface contact resistance in the MTLM sample (from the CIGS solar module) and in the i-TLM sample. The temperature dependence of the contact resistance was characterized by measuring the $\mathrm{I}-\mathrm{V}$ characteristics at various temperatures between $100 \mathrm{~K}$ and $300 \mathrm{~K}$.

Calculation of the series resistance due to the TCO layer. Current flows in the solar cell devices are generally perpendicular to the $\mathrm{p}-\mathrm{n}$ junction. To extract current via contacts, the current must flow laterally along the TCO window layer. The series resistance due to the TCO window layer could be calculated using an approach similar to that used to calculate the power loss in a reference ${ }^{15}$, based on the calculation of the voltage drop imposed by the lateral current flow along the TCO layer. Assuming that the photocurrent density or dark current density flowing through the p-n junction of the solar cell was uniform over the active cell area, the lateral current in the TCO layer a distance $(\mathrm{x})$ away from the collecting contact was obtained by integrating the current density through the pn junction (J) from the position (x) to the outer edge of the cell (b), as follows:

$$
\mathrm{I}_{\mathrm{TCO}}(\mathrm{x})=\int_{\mathrm{x}}^{\mathrm{b}} \mathrm{Jdx}=\mathrm{J}(\mathrm{b}-\mathrm{x})
$$

where $\mathrm{J}$ is the current density flowing through the CdS/CIGS junction, and $\mathrm{b}$ is the width of the Mo electrode finger in the module. Whereas the currents collected into the TCO layer travelled laterally along the TCO layer, the voltage drop $\left(\mathrm{V}_{\mathrm{TCO}}\right)$ could be obtained from the product of the current distribution $\left(\mathrm{I}_{\mathrm{TCO}}\right)$ and the incremental resistance $(\mathrm{dV}=\mathrm{IdR})$, as follows:

$$
\mathrm{V}_{\mathrm{TCO}}=\int_{0}^{\mathrm{b}} \mathrm{I}_{\mathrm{TCO}}(\mathrm{x}) \mathrm{dR}=\int_{0}^{\mathrm{b}} \mathrm{I}_{\mathrm{TCO}}(\mathrm{x}) \frac{\rho_{\mathrm{TCO}}}{\mathrm{t}} \mathrm{dx}=\mathrm{J} \frac{\rho_{\mathrm{TCO}}}{\mathrm{t}} \frac{\mathrm{b}^{2}}{2},
$$

where $\rho_{\mathrm{TCO}}$ is the electrical resistivity of the TCO layer and $\mathrm{t}$ is the layer thickness. The resultant contribution to the series resistance is given by:

$$
\mathrm{R}_{\mathrm{S}, \mathrm{TCO}}=\frac{\mathrm{V}_{\mathrm{TCO}}}{\mathrm{J}}=\frac{\rho_{\mathrm{TCO}} \mathrm{b}^{2}}{2 \mathrm{t}} .
$$

1. Chirilă, A. et al. Potassium-induced surface modification of $\mathrm{Cu}(\mathrm{In}, \mathrm{Ga}) \mathrm{Se}_{2}$ thin films for high-efficiency solar cells. NAT. MATER. 12, 1107-1111 (2013).

2. Jackson, P., Hariskos, D., Wuerz, R., Wischmann, W. \& Powalla, M. Compositional investigation of potassium doped $\mathrm{Cu}(\mathrm{In}, \mathrm{Ga}) \mathrm{Se}_{2}$ solar cells with efficiencies up to $20.8 \%$. Phys. Status Solidi-Rapid Res. Lett. 8, 219-222 (2014).

3. Rau, U. \& Schock, H. W. [Cu(In,Ga)Se $e_{2}$ Solar Cells] Clean Electricity from Photovoltaics [Archer, M. D. \& Hill, R. (ed.)] [301-306] (Imperial College Press, London, 2001).

4. Shafarman, W. N., Siebentritt, S. \& Stolt, L. [Cu(InGa)Se 2 Solar Cells] Handbook of Photovoltaic Science and Engineering [Luque, A. \& Hegedus, S. (ed.)] [583-590] (John Wiley and Sons, Chichester, 2011).

5. Klaer, J. et al. Damp heat stability of chalcopyrite mini-modules: evaluation of specific test structures. Proc. $31^{\text {st }}$ IEEE PVSC, 336-339; DOI:10.1109/ PVSC.2005.1488137 (2005).

6. BERGER, H. H. Models for Contacts to Planar Devices. Solid-State Electron. 15, 145-158 (1972).

7. Schroder, D. K. Semiconductor Material and Device Characterization. [127-184] (John Wiley and Sons, Hoboken, 2006).

8. Hegedus, S. S. \& Shafarman, W. Thin-film solar cells: device measurements and analysis. Prog. Photovoltaics 12, 155-176 (2004).

9. Kohara, N., Nishiwaki, S., Hashimoto, Y., Negami, T. \& Wada, T. Electrical properties of the $\mathrm{Cu}(\mathrm{In}, \mathrm{Ga}) \mathrm{Se}_{2} / \mathrm{MoSe}_{2} / \mathrm{Mo}$ structure. Sol. Energy Mater Sol. Cells 67, 209-215 (2001).

10. Shafarman, W. \& Phillips, J. Direct current-voltage measurements of the Mo/ $\mathrm{CuInSe}_{2}$ contact on operating solar cells. Proc. $25^{\text {th }}$ IEEE PVSC, 917-919; DOI:10.1109/PVSC.1996.564278 (1996).

11. Yoon, J.-H. et al. Electrical properties of CIGS/Mo junctions as a function of $\mathrm{MoSe}_{2}$ orientation and Na doping. Prog. Photovoltaics 22, 90-96 (2013).

12. Abou-Ras, D. et al. Formation and characterisation of $\mathrm{MoSe}_{2}$ for $\mathrm{Cu}(\mathrm{In}, \mathrm{Ga}) \mathrm{Se}_{2}$ based solar cells. Thin Solid Films 480, 433-438 (2005).

13. Caballero, R. et al. Influence of $\mathrm{Na}$ on $\mathrm{Cu}(\mathrm{In}, \mathrm{Ga}) \mathrm{Se}_{2}$ solar cells grown on polyimide substrates at low temperature: Impact on the $\mathrm{Cu}(\mathrm{In}, \mathrm{Ga}) \mathrm{Se}_{2} / \mathrm{Mo}$ interface. Appl. Phys. Lett. 96, 092104 (2010).

14. Kohara, N., Negami, T., Nishitani, M. \& Wada, T. Preparation of device-quality $\mathrm{Cu}(\mathrm{In}, \mathrm{Ga}) \mathrm{Se}_{2}$ thin films deposited by coevaporation with composition monitor. Jpn. J. Appl. Phys. 34, L1141-L1144 (1995).

15. Green, M. A. Solar Cells: Operating Principles, Technology, and System Applications [145-147] (Prentice-Hall Inc, Englewood Cliffs, 1982).

\section{Acknowledgments}

This work was supported by a KIST internal project and partially by the New \& Renewable Energy Technology Development Program under a grant from the Korea Institute of Energy Technology Evaluation and Planning (KETEP) (No. 20113020010050), funded by the Ministry of Trade, Industry \& Energy (MOTIE), and the financial support from "Development of 25\% efficiency grade tandem CIGS thin film solar cell core technology (B551179-12-01-00)“ of Korea Research Council for Industrial Science and Technology (ISTK).

\section{Author contributions}

J.-H.Y. performed the experiments of sample fabrication and measurement. J.-K.P. and W.M.K. participated in the interpretation of data and the critical revision of the manuscript. J.L. and H.P. provided the searched publication to help come up with the original idea, and 
prepared the solar modules at LG Innotek. J.-h.J. conceived the original idea, designed the study, and wrote the manuscript.

\section{Additional information}

Competing financial interests: The authors declare no competing financial interests.

How to cite this article: Yoon, J.-H. et al. Characterization of efficiency-limiting resistance losses in monolithically integrated $\mathrm{Cu}(\mathrm{In}, \mathrm{Ga}) \mathrm{Se}_{2}$ solar modules. Sci. Rep. 5, 7690; DOI:10.1038/srep07690 (2015).
(1) This work is licensed under a Creative Commons Attribution-NonCommercialNoDerivs 4.0 International License. The images or other third party material in this article are included in the article's Creative Commons license, unless indicated otherwise in the credit line; if the material is not included under the Creative Commons license, users will need to obtain permission from the license holder in order to reproduce the material. To view a copy of this license, visit http:// creativecommons.org/licenses/by-nc-nd/4.0/ 